\title{
Design and Implementation of Laboratory Information Management System for Chemical Analysis
}

\author{
LI Qinghua, a \\ ${ }^{1}$ Nanchang Institute of Science and Technology, Nanchang 330108,China \\ aliqinghua@126.com
}

Keywords: Laboratory information management system; Chemical analysis laboratory; B/S architecture; PHP; Zend Framework; Oracle database

\begin{abstract}
.
With the development and deepening of the scientific research field, as well as the more and more comprehensive and standardized laboratory management, the business data and the management data of the laboratory are increasing with the geometric progression. How to effectively record, manage and utilize these data resources has become an important problem to be solved urgently. If only by artificial means of classification, sorting and analysis, it is obviously unscientific and unrealistic. Today the information technology development is increasingly mature, the establishment of laboratory information management system to realize the sharing of all kinds of laboratory quality data collection, processing, fast transmission, and storage, is of great significance for the development of laboratory.
\end{abstract}

\section{Introduction}

\subsection{The concept of laboratory information management system}

With the sustained and rapid development of China's national economy and the continuous improvement of people's living standards, the public's attention to product quality, food safety, health and quarantine has reached an unprecedented height. In our country, all kinds of testing laboratory has been popping up like bamboo shoots after a spring rain, such as: Quality Supervision and inspection of import and export commodities, disease prevention and control, environmental monitoring, agricultural products inspection and other large industrial enterprises and other specialized laboratories in the national economy important units are equipped with large scale analysis and testing laboratory as test data the provider will play a crucial role. [1]With the continuous development of scientific research and production technology, the original manual management mode is not adapt to the requirements of people, both in the analysis and test of sample number and cycle analysis and analysis are put forward higher requirements of project and data authority, reliability, laboratory information management system (LIMS) is produced in this a background.

LIMS is specially applied in the testing laboratory analysis all kinds of information, especially the analysis of information network management system samples. It can be for the laboratory efficient and scientific operation and the preservation of information, communication and processing platform of quantitative quality control and all aspects of laboratory work; it is the combination of computer technology management science and technology analysis. It sets the analysis of modern management theory and computer technology, technology for a new application 
of the laboratory management and control, can greatly improve the laboratory management level and management efficiency. At present, LIMS has been widely used in developed countries, while the country is still in the initial stage of development and application.

\subsection{Market demand forecast}

In China, the LIMS system has a wide range of applications, suitable for analysis and testing laboratory of pharmaceutical, petroleum and chemical industry, environmental protection, water supply, medical, mining, metallurgy, quarantine, customs, tobacco, wine, beverage, food, commodity inspection, power and education and scientific research units and other industries.

According to the statistics of the Ministry of science and technology, at present our country is engaged in the detection mechanism for the community to provide impartial data about a total of more than 20 thousand seized product categories covering our product categories a total of $98.8 \%$, including the quality supervision agencies at all levels of the quality inspection institute; inspection agencies at all levels of the entry-exit inspection and quarantine agencies are set up respectively; the industry department quality inspection center (including the National Center and ministerial Center), quality inspection station; authorized in the work of the enterprise quality inspection laboratory. As the main body of China's social detection mechanism is part of the quality inspection department shall set up by law, and authorized by the Department to set up all kinds of quality inspection agencies at all levels, a total of more than 5 thousand and 500 employees, about 11 million, net fixed assets of nearly ten billion yuan, has about 330 thousand sets of equipment. [2]As of January 31, 2006, the CNAL approved laboratory has reached 2431, of which the testing laboratory of 2123. How to regulate the operation of laboratory personnel, equipment and business processes, to better meet the laboratory accreditation requirements, to win public trust, improve the visibility of the laboratory, which is a issuse that laboratory must be considered.

\section{Related technology to laboratory information management system}

\subsection{UML profile}

The UML language is used to describe the software, visualize the process, construct and build the document of the software system. It records the decision and understanding of the system to be constructed, and can be used to understand, design, configure, maintain and control information.

With the development of programming language, object-oriented programming thought is more and more recognized by the person, which has become the mainstream of the program design method. At the same time oriented object analysis and design (OOA/OOD) methods are gradually deepening. Research results varied, the emergence of a variety of ideas and methods for analysis of the object system, it also brings some problems: various methods of surface objects have many similar concepts, definitions and descriptions with each other but not the same, the error between the different methods in understanding, especially in the symbol.[3] This situation creates technical communication difficulties, but also is not conducive to new users to choose a stable modeling tool to help the system analysis and design.

Therefore, it is urgent to set up a set of unified modeling methods on the basis of these research results. The advantage of unified modeling language is that it can form a unified system analysis model and expression method, which is conducive to the mutual learning between the systems and improve the system design ideas. And on the basis of unified modeling, we can continue to improve this method, which is beneficial to everyone's acceptance, which is the inevitable trend of the development of system analysis methods.

The main content of UML mainly includes two aspects, namely UML semantics and UML representation. 
UML semantics is used to describe the definition of Meta model. UML provides a simple, consistent and general definition of all elements to avoid ambiguity and ambiguity.

UML notation defines UML symbolic language. This set of symbols unifies the graphical symbols used in the modeling language. This symbol is used to represent the application level model.

\subsection{RUP profile}

Unified software process (RUP) is a set of software process method, which absorbing the advantages of a variety of development models, and of good operability and practicality. At the same time, RUP is a set of software engineering methods, each organization can according to their own actual situation, as well as the scale of the project to the RUP cutting and modification, in order to develop the software engineering process.

RUP is an iterative and incremental development process driven by use case via architecture centric.

\subsubsection{Drinven by use case}

The software system is designed to serve its users. Therefore, in order to construct a successful software system, it is necessary to understand what the user wants and needs. The user represents a person or thing that interacts with the developed system. Users meet their own needs through a series of interaction with the system. This interaction is the use case; the system is able to provide users with valuable results of a function. All cases are combined to form the use case model, and the use case is not only a tool to determine the system requirements, but also can drive the design, implementation and test of the system. [4]Use case is the basis of analysis and design of if, the developer creates a series of design model and implementation model to implement the use case. And then the use case model is usded to review and test the implementation of the system. The development process by case, cases were identified, along the cases are designed to be implemented by the workflow, and use case testing case, not only start the development process, and make the combination as a whole, therefore, it can be said that the RUP process is use case driven.

\subsubsection{Architecture-centric}

The architecture of the software system describes the system to be constructed from different angles, and describes the overall design of the system. Each product has its function and form two, function and corresponding case here, form and structure corresponding to form here not only refers to the external manifestation of the software, but also refers to a kind of from inside to outside the structure form of functions. The use case and the framework affect each other, on the one hand, the use case must be suitable for the architecture in the implementation; on the other hand, the framework must be reserved for the purpose of realizing the present or future use cases. From the understanding of the main functions of the system, the architect must extract the core use cases, design the framework of evolution, construct the use cases around the system framework, and develop the system.

\section{Design of the system}

\subsection{Design of the system architecture}

Through the investigation and the demand of the system environment of the early analysis, considering all the objective factors, we determine the system will use the B/S (Browser/Server, browser / server) software architecture and the three layer system structure.

\subsubsection{Brief introduction of B/S structure}

The structure of $\mathrm{B} / \mathrm{S}$ is composed of three parts: the client, application server and database server, which is the improvement of the structure of a kind of change of C/S (Client/Server). It does not need to install special client software on the client, the user access the Web server to achieve 
through the browser program, a small part of the transaction logic in the front, but the main business logic on the server, forming a 3 layer structure (Web/Server/Database).

Compared with the traditional C/S application system structure of two layers, three layers B/S architecture application client function is greatly reduced, only to achieve human-computer interaction, application logic, the original by the client to achieve the data access functions are migrated to the application server layer to achieve, so the client usually called it "thin client".

\subsubsection{Architecture of software}

In order to adapt and cooperate with the B/S running mode of the system, the system adopts the multi-layer software architecture based on the Zend framework, including the presentation layer, the business logic layer and the data access layer as shown in Figure 1. This kind of multi-layer architecture can realize the dynamic and sustainable program design, so that the subsequent modification and extension of the program is simplified, and it is widely used in the three tier architecture.

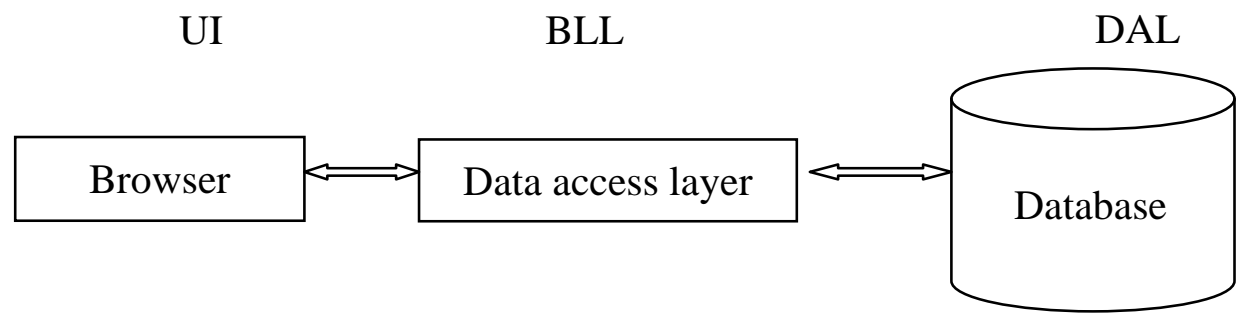

Figure 1. Three-tier system structure diagram

\subsection{Design of function structure}

According to the analysis results of the laboratory business model, and the laboratory information management function, the system will be divided into nine main modules, respectively, experimental process management, equipment management, materials management, document management, personnel management, project management, information, system management and login authentication module.

\subsection{Design of database}

System database design is based on the needs of users, the real world within a certain range of data abstracted into the specific structure of the database process. A good database structure is conducive to the development and maintenance of the entire system, the system uses the relational database model, the use of Oracle database management system.

\subsubsection{Data model}

Hierarchical model, network model and relational model are three traditional data models. The hierarchical model with tree structure to identify links between entities, but the hierarchical model cannot represent many to many relationships; network model with the network structure that links between entities, but this model in data independence is still great deficiency; the relational model is that contact data model entities and entities in the form of two-dimensional, a rigorous mathematical basis, relatively high level of abstraction, and simple data model is more popular now, and most database systems support this model.

\subsubsection{Data dictionary}

With the data model of the system, we can design the data dictionary of the system. Based on the system model, we can accord the characteristics of Oracle database management system; determine the storage structure of the database, including the data field of the storage type, size, default and restriction. The system database table and the relationship is basically one-to-one, in addition, we also created several database tables, for some function support system, to maintain the normal 
operation of the system, this system including the announcement information table, remind the user information table, user information table, role information table, system resource table system and constant information table etc.. [5]Because of the structure of these data sheets are relatively simple, this is no longer a detailed description of the system data dictionary details see Appendix "database physical structure table".

\subsubsection{Security of system design}

The safety protection measures of laboratory information management system can be divided into two categories: non-technical and technical. Non-technical safety measures mainly refer to the administrative management, rules and regulations and other physical measures, which has nothing to do with the design of the system. Technology security measures mainly refers to the use of technical means and system in the internal system, to prevent unauthorized access and use of system resources, ensure the security and integrity of system data, and maintain the stability of system running. In the design of the system, we mainly take the following two measures to ensure the security of the system: user authentication and access control, and data backup and recovery.

\section{References}

With the rapid development of the domestic economy, people's demand for life is getting higher and higher, and the demand for a variety of food is also growing. At the same time, with the growth of economy, the increase of people's income, the improvement of living standard and the quality of life, people pay more attention to the quality of all kinds of food and supplies. In recent years, a variety of domestic food problems emerge in an endless stream, so more people pay attention to the safety of all kinds of food and supplies. With people's increasing attention to all kinds of product quality, various domestic laboratories continue to emerge. The laboratory not only undertakes a variety of quality supervision, inspection and testing of import and export commodities, food safety and sanitation and other tasks, but also plays an important role for environmental monitoring, agricultural products detection. With the increase of the number of samples in various testing laboratories, the use of manual management of samples has been completely unable to meet the needs of various types of laboratory management. The introduction of laboratory management information system can not only improve the efficiency of laboratory management, but also can improve the reliability and authority of all kinds of test reports and test data, thus improve the accuracy of testing data.

\section{Summary}

[1] Fu Xuesheng. The latest progress and development trend of LIMS in the world [J]. Modern scientific instrument.2013, 17-20.

[2] Guo Baohong, Sun Jing. Research and development of open computer laboratory management system [J]. Tianjin Journal of science and technology, 2014, 73-76.

[3]Eric Armstrong, Jennifer Ball, Stephanie Bodoff [M]. The Web Thtoria California.2013, 7:23-25. [4]Joshua Bloch. Java programming guide [M]. Tianjin: China Electric Power Press, (in Chinese) language, 2014, 202-208.

[5]The Husted, Cedric Dumoulin, George Franciscus, etal. Struts Action: Building Applications with Leading Jaba Framework[M]. Manning Publications, 2014, 33-34. 